\title{
Erector spinae block for postoperative analgesia following axillary hidradenitis suppurativa resection: a case report
}

This article was published in the following Dove Press journal: Local and Regional Anesthesia

\section{Johanna Blair de Haan Nadia Hernandez Sudipta Sen}

Department of Anesthesiology, McGovern Medical School, University of Texas Health Science Center at Houston, Houston, TX 77030, USA
Correspondence: Johanna Blair de Haan Department of Anesthesiology,

McGovern Medical School, University of Texas Health Science Center at Houston, 643I Fannin St., MSB 5.020, Houston, TX 77030, USA

Tel +I 36I 5634664

Email johanna.b.dehaan@uth.tmc.edu

\begin{abstract}
Hidradenitis suppurativa (HS) is a chronic inflammatory disease of the terminal hair follicle of the apocrine gland-bearing skin, presenting with inflamed nodules, abscesses, and sinus tracts. Autoimmune, genetic, and infectious factors have been implicated in its pathogenesis. HS can be managed medically using topical and systemic antibiotics and with tumor necrosis factor antibody therapy in severe cases. Surgical treatment can range from local excision, deroofing or excision of sinus tracts, or wide radical excision of severe lesions. Lesions can be severely painful on their own or following surgical resection. Patients may require opioid therapy due to pain from the lesions themselves or following painful surgical resection. Erector spinae block (ESB) is a recently developed plane block used for the management of pain located in the chest wall or the abdominal wall. Ultrasound guidance is used to guide placement of the needle tip between the transverse process at the desired dermatomal level and the erector spinae muscle group. Traditional descriptions of the ESB have been at the level of the fifth transverse process to treat chest wall pain; in this case report, we describe a novel use of ESB at the level of the second transverse process to treat axillary pain after surgical debridement of axillary HS.
\end{abstract}

Keywords: erector spinae block, hidradenitis suppurativa, axillary pain, truncal blocks, ultrasound-guided blocks, axillary analgesia, thoracic analgesia, thoracic wall block, peripheral nerve block

\section{Introduction}

The erector spinae block (ESB) has gained popularity recently for its usefulness in managing chest wall and upper abdominal pain. It has been used for managing pain after sternotomies, mastectomies, bariatric surgeries, abdominal surgeries, rib fractures, and thoracotomies. ${ }^{2-6}$ Autoimmune, genetic and infectious factors have been implicated in the pathogenesis of hidradenitis suppurativa (HS). ${ }^{1} \mathrm{HS}$ can be managed with either topical or systemic antibiotics, or tumor necrosis factor (TNF) antibody therapy in severe cases. ${ }^{1,11}$

Pain due to HS has both acute and chronic components. The acute component is inflammatory in nature and is related to the inflamed cysts and nodules that develop. ${ }^{9,10,12,13}$ Patients describe it as being similar to neuropathic pain, citing sensations of stabbing, burning, and shooting. According to Patel et al, ${ }^{12}$ chronic pain is associated with more advanced inflammatory HS, and is described by patients as aching, throbbing, and gnawing. ${ }^{13}$ Another study, by Kofler et al, showed that the incidence of postoperative pain following excision of HS lesions occurred in $65 \%$ of patients, with $38 \%$ of patients requiring analgesics. ${ }^{14}$

We present a case wherein the ESB was used to successfully treat axillary pain following resection of axillary HS. Written informed consent was obtained from the 
patient to report findings and publish this case report, including images obtained of the area of analgesia. Institutional approval was not required for publication of this case report.

\section{Case report}

The patient is an otherwise healthy, 18-year-old male with a body mass index of $37 \mathrm{~kg} / \mathrm{m}^{2}$, who presented with chronic bilateral axillary, bilateral thigh, and posterior neck HS lesions. He had failed medical treatment including injectable tumor necrosis factor antibody therapy and presented for wide radical excision of his axillary lesion with a plan for later skin grafting by plastic surgery.

He was previously treated with adalimumab (Humira), a tumor necrosis factor antagonist, without resolution. He was brought to the operating room for excision of his right axillary lesions. General anesthesia was induced with 4 $\mathrm{mg}$ midazolam, $150 \mu \mathrm{g}$ fentanyl, $80 \mathrm{mg}$ lidocaine, $200 \mathrm{mg}$ propofol, and $50 \mathrm{mg}$ rocuronium. An $8.0 \mathrm{~mm}$ endotracheal tube was inserted through the vocal cords. Anesthesia was maintained using oxygen, air, and sevoflurane. Additional $400 \mathrm{mg}$ propofol, $20 \mu \mathrm{g}$ dexmedetomidine, and $100 \mu \mathrm{g}$ fentanyl were administered in total during the surgery. Patient received $1 \mathrm{~g}$ intravenous acetaminophen, $30 \mathrm{mg}$ ketorolac, and $1 \mathrm{mg}$ hydromorphone for pain control. Four milligrams of ondansetron and $4 \mathrm{mg}$ of dexamethasone were administered, and the patient was extubated successfully after reversal of paralytic with $4 \mathrm{mg}$ of neostigmine and $0.8 \mathrm{mg}$ of glycopyrrolate. Surgery involved excision of skin and subcutaneous tissue of the axilla and placement of a negative pressure wound vacuum.

The surgery team requested the regional anesthesia team to perform a block to control postoperative pain, as axillary resection of the soft tissue was extensive. Prior to placement of ESB, patient was awakened from general anesthesia and he recovered in the post-anesthesia care unit (PACU). He was evaluated by the regional anesthesia team 37 minutes after his arrival to the PACU, at which time he was fully awake and oriented to person, place, and time, and his self-reported pain score was 8 on the numeric rating scale.

We performed an ESB in the PACU, with the patient lying in left lateral decubitus position. The patient's back was palpated for landmarks; the seventh cervical spinous process (C7) was identified and marked, and used to count caudally to thoracic vertebra 2 (T2). The T2 spinous process was identified and marked. A high-frequency linear transducer (HFL50; 15-6 MHz) was placed on the back, slightly to the right of midline in a coronal plane. The transducer was translated medially until the transverse processes (TPs) were identified, and then cephalad to identify the T2 TP, lateral to the marked T2 spinous process. Skin was then sterilized with ChloraPrep ${ }^{\mathrm{TM}}$ skin prep solution $(2 \% \mathrm{w} / \mathrm{v}$ chlorhexidine gluconate in $70 \% \mathrm{v} / \mathrm{v}$ isopropyl alcohol; BD, Franklin Lakes, NJ, USA), and a 20-gauge, blunt-tipped, $100 \mathrm{~mm}$ echogenic block needle (B Braun, Bethlehem, PA, USA) was advanced in-plane from caudal to cranial to make osseous contact with the T2 TP. At this point, a small volume of saline was injected to assess for satisfactory spread of fluid underneath the erector spinae muscle (ESM) and above the TP. A volume of $30 \mathrm{~mL}$ of $0.25 \%$ bupivacaine hydrochloride ( $75 \mathrm{mg}$ ) with $3 \mathrm{mg}$ of preservative-free dexamethasone was then injected under direct ultrasound visualization (Sonosite X-Porte; SonoSite, Bothell, WA, USA). Craniocaudal spread was noted below the ESM (Figure 1).

Following block placement, he had a marked decrease in axillary pain, from the aforementioned pain score of 8 to 0 on the numeric rating scale, 25 minutes after completion of the ESB. He did not require any narcotics or adjuncts for pain in the recovery room. He was maintained on scheduled acetaminophen $1 \mathrm{~g}$ every 6 hours, celecoxib 200mg every 12 hours, and gabapentin $300 \mathrm{mg}$ every 8 hours. Tramadol 100mg was given every 6 hours as needed for breakthrough pain. Neurological assessment to pin prick was performed, and diminished sensation was noted on the right side over the shoulder, scapula, axilla, and anterolateral chest wall (Figures 2 and 3). Handgrip, flexion and extension at wrist and elbow, and abduction and adduction at the shoulder were intact.

The patient underwent two more debridements of the axilla and placement of negative pressure wound vacuum at days 3 and 6 after his initial surgery under general anesthesia.

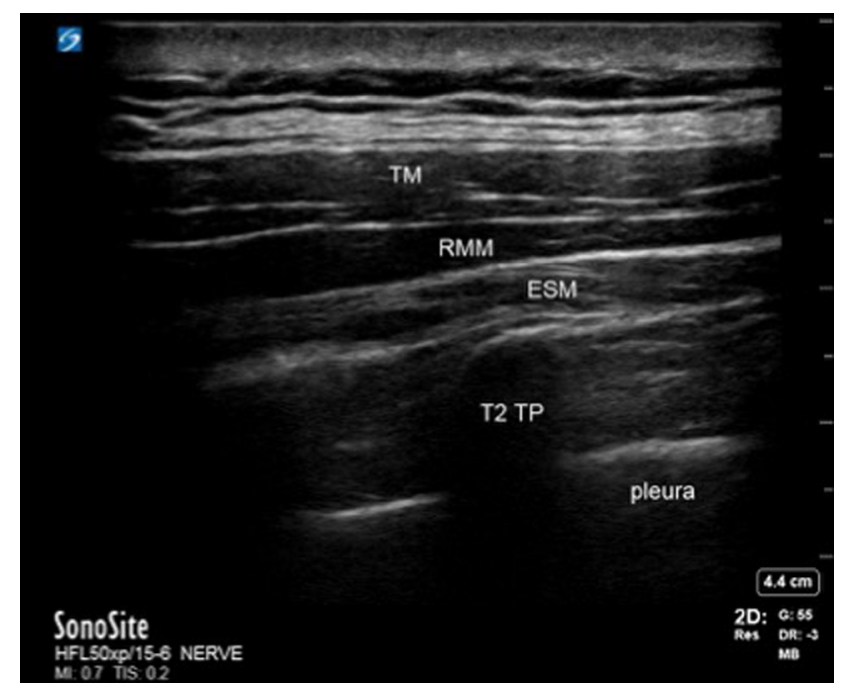

Figure I Ultrasound image of the three muscle layers.

Notes: TM, RMM, and ESM overlying the T2 TP. LA spread is seen in between the ESM and T2 TP.

Abbreviations: ESM, erector spinae muscle; LA, local anesthetic; RMM, rhomboid major muscle; TM, trapezius muscle; TP, transverse process. 


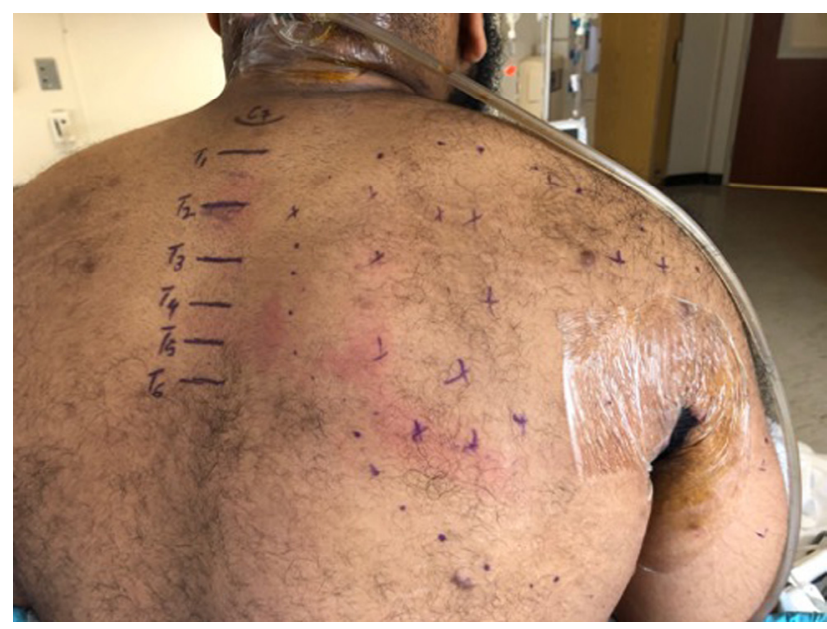

Figure 2 Extent of diminished sensation to pin prick on postoperative day I; right shoulder and right scapula.

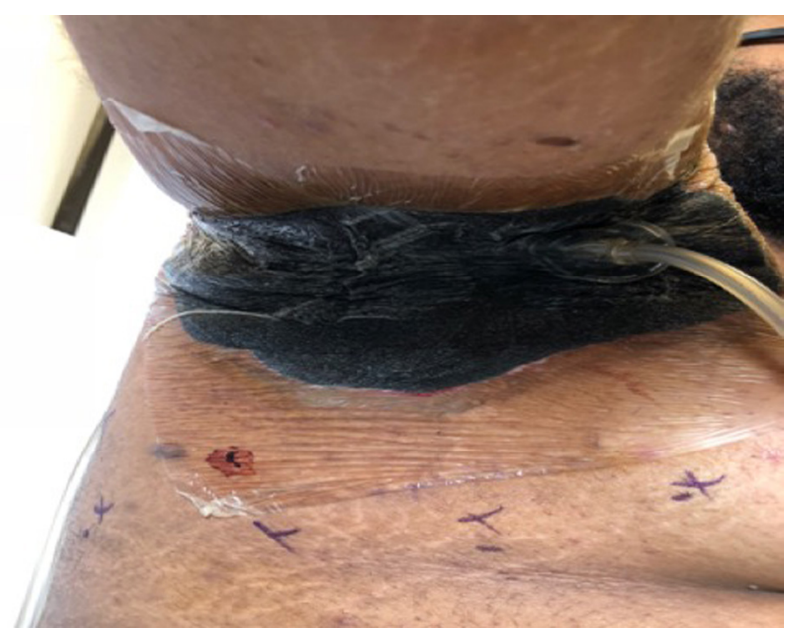

Figure 3 Extent of diminished sensation to pinprick on postoperative day I; right axilla and right anterolateral chest wall.

He requested the regional team perform the same block, as he was satisfied with the analgesia. ESB was performed in a similar fashion as described above with similar analgesic efficacy. Average time to first narcotic consumption after each T2 ESB was 39.3 hours.

This patient was receiving chemical venous thromboembolic (VTE) prophylaxis, beginning after his first surgery. He was receiving scheduled enoxaparin $40 \mathrm{mg}$ once daily at the time of the second and third ESB.

\section{Discussion}

ESB was first described as a paraspinous interfacial regional anesthesia technique that deposits local anesthetic (LA) below the ESM and above the T5 TP. The extent of analgesia is secondary to diffusion of the LA into the paravertebral space at multiple levels blocking the ventral and dorsal ramii of the spinal nerves. Spread of iodinated contrast dye was seen between $\mathrm{T} 2$ and T8 when $20 \mathrm{~mL}$ of contrast was injected at T5 in fresh cadavers deep to the ESM. ${ }^{3}$

In this case report, we performed ESB at T2 TP with 30 $\mathrm{mL}$ of LA to cover the upper thoracic dermatomes supplying the axilla and the anteriolateral and posteriolateral chest wall. As the ESM is a group of three muscles, spinalis (thoracis and cervicis), longismus, and iliocostalis, which extends from the sacrum and lumbar spine to the thoracic and cervical spine, we reasoned that performing this interfacial plane block at T2 TP would provide optimal spread of LA to cover the axilla. We provided T1-T10 dermatomal analgesia with ESB to an infected area with minimal risk of needle site infection due to the distant needle entry point. This patient consistently demonstrated good sensory analgesia with this technique, resulting in minimal narcotic consumption and early rehabilitation. Lack of motor deficit was presumed to be due to the high $\mathrm{pKa}$ of $0.25 \%$ bupivacaine hydrochloride, which decreases the rate of penetration into the large myelinated A fibers which are responsible for motor conduction. ${ }^{7}$

ESB has a better safety profile than paravertebral nerve block as the risk of pleural puncture and epidural spread is minimal. A theoretical risk of phrenic nerve paresis exists while performing a high ESB. Forero et al demonstrated minimal spread of radiocontrast dye to the cervical nerve roots in a patient with chronic shoulder pain who received an ESB at T2 level. ${ }^{8}$ Our patient did not experience symptomatic phrenic nerve block. The second and third ESBs on this patient were performed post-induction of general anesthesia and he was extubated without any complications.

Another advantage of ESB over paravertebral nerve block is the ability to perform ESB without timing limitations by chemical VTE prophylaxis. This patient was receiving once daily enoxaparin $40 \mathrm{mg}$ at the time of his second and third ESB, and did not develop a hematoma. Chemical VTE prophylaxis is scheduled and ordered for all patients without contraindication at our institution, per facility protocol, due to our high incidence of thromboembolic events. The site on the patient's back was examined daily by the acute pain service and was not found to be tender, enlarged, or to have evidence of bleeding. During subsequent blocks, anatomy was unchanged by swelling: there was no ultrasonographic evidence of hematoma.

The dermatomes to be covered could also have been blocked via individual intercostal nerve blocks. However, injection of LA at multiple intercostal levels can result in LA toxicity as the uptake of LA at this site is high due to increased vascularity. This also shortens the block duration due to the speed with which the LA is absorbed and metabolized. The 
ESB is an avascular plane block which allows for a longer duration of action of LA, and results in lower plasma volumes due to reduced uptake of the LA.

Brachial plexus blockade would not anesthetize the axilla because the axilla is innervated by the T2 intercostal nerve, which is not a part of the brachial plexus. A high thoracic epidural could also have been performed in order to alleviate axillary pain; however, this would have placed him at risk for hypotension and bradycardia due to blockade of the cardiac accelerator fibers. ESB provided a technique to alleviate his pain without the added sympathectomy of a neuraxial technique.

ESB is a valuable resource, and regional anesthesiologists should be creative with their uses of this block. It is a versatile technique, as evidenced by its application at various spinal levels in unique surgical procedures.

\section{Disclosure}

The authors report no conflicts of interest in this work.

\section{References}

1. WoodruffCM, Charlie AM, Leslie KS. Hidradenitis suppurativa: a guide for the practicing physician. Mayo Clin Proc. 2015;90(12):1679-1693.

2. Chin KJ, Malhas L, Perlas A. The erector spinae plane block provides visceral abdominal analgesia in bariatric surgery: a report of 3 cases. Reg Anesth Pain Med. 2017;42(3):372-376.
3. Forero M, Adhikary SD, Lopez H, Tsui C, Chin KJ. The erector spinae plane block: a novel analgesic technique in thoracic neuropathic pain. Reg Anesth Pain Med. 2016;41(5):621-627.

4. Hamilton DL, Manickam B. Erector spinae plane block for pain relief in rib fractures. Br J Anaesth. 2017;118(3):474-475.

5. Hannig KE, Jessen C, Soni UK, Børglum J, Bendtsen TF. Erector spinae plane block for elective laparoscopic cholecystectomy in the ambulatory surgical setting. Case Rep Anesthesiol. 2018;2018:5492527.

6. Kimachi PP, Martins EG, Peng P, Forero M. The erector spinae plane block provides complete surgical anesthesia in breast surgery: a case report. A A Pract. 2018;11(7):186-188.

7. Wildsmith JA, Brown DT, Paul D, Johnson S. Structure-activity relationships in differential nerve block at high and low frequency stimulation. Br J Anaesth. 1989;63(4):444-452.

8. Forero M, Rajarathinam M, Adhikary SD, Chin KJ. Erector spinae plane block for the management of chronic shoulder pain: a case report. Can J Anaesth. 2018;65(3):288-293.

9. Kurzen H, Kurokawa I, Jemec GB, et al. What causes hidradenitis suppurativa? Exp Dermatol. 2008;17(5):455.

10. Saygin D, Syed AU, Lowder CY, Srivastava S, Maya JJ, Hajj-Ali RA. Characteristics of inflammatory eye disease associated with hidradenitis suppurativa. Eur J Rheumatol. 2018;5(3):165-168.

11. Saunte DML, Jemec GBE. Hidradenitis suppurativa: advances in diagnosis and treatment. JAMA. 2017;318(20):2019-2032.

12. Patel ZS, Hoffman LK, Buse DC. Disability, and impaired quality of life in hidradenitis suppurativa. Curr Pain Headache Rep. 2017;21(12):49.

13. Horváth B, Janse IC, Sibbald GR. Pain management in patients with hidradenitis suppurativa. J Am Acad Dermatol. 2015;73(5 Suppl 1): S47-S51.

14. Kofler L, Schweinzer K, Heister M, Kohler M, Breuninger H, Häfner HM. Surgical treatment of hidradenitis suppurativa: an analysis of postoperative outcome, cosmetic results and quality of life in 255 patients. J Eur Acad Dermatol Venereol. 2018;32(9):1570-1574.
Local and Regional Anesthesia

\section{Publish your work in this journal}

Local and Regional Anesthesia is an international, peer-reviewed, open access journal publishing on the development, pharmacology, delivery and targeting and clinical use of local and regional anesthetics and analgesics. The journal is included in PubMed, and welcomes submitted papers covering original research, basic science, clinical studies,

\section{Dovepress}

reviews and evaluations, guidelines, expert opinion and commentary, case reports and extended reports. The manuscript management system is completely online and includes a very quick and fair peer-review system, which is all easy to use. Visit http://www.dovepress.com/ testimonials.php to read real quotes from published authors. 